\title{
Analysis Of "Invisible" Poly-Bump Defect With EFTEM Spectral Imaging
}

\author{
C.T. Schamp, ${ }^{*}$ R. Rios, ${ }^{* *}$ A. Gill* \\ *Cerium Laboratories ${ }^{\mathrm{TM} \dagger}, 5204$ E. Ben White Blvd. MS512, Austin, TX 78741 \\ **Spansion Inc., 915 Deguigne Dr., PO Box 3453, Sunnyvale, CA 94088
}

Polycrystalline Si layers are often used in the production of Si-based semiconductor devices [1]. These layers can exhibit defects typically called "poly-bumps" or "poly-nodules" in which an analysis usually involves recording cross-section SEM and/or TEM bright-field micrographs that often show no apparent cause for the bump on the poly-Si surface [2]. In this extended abstract, analysis beyond typical bright-field TEM imaging is presented showing an embedded particle giving raise to excess growth of the poly-Si leading to a bump on the surface.

Through the use of a current generation dual-beam FIB instrument, specifically an FEI Expedia 1255 using $30 \mathrm{kV}$ bulk milling, $5 \mathrm{kV}$ fine slicing, and a $2 \mathrm{kV}$ clean-up step; cross-section TEM samples on the order of $50-80 \mathrm{~nm}$ thick can be routinely prepared. By carefully milling material away on either side of the defect to the point where the defect becomes visible through the SEM imaging system on both sides of the lamella, one can be certain of encapsulating the defect in the sample. An SEM image shown in figure 1 confirming the presence of a defect was acquired using an Hitachi 4800 SEM at $2 \mathrm{kV}$. The TEM analysis presented here was carried out using a Philips/FEI CM300 (S)TEM operated at $300 \mathrm{kV}$. This TEM is equipped with a model 2002 Gatan Imaging Filter which was used to acquire Energy Filtered TEM (EFTEM) data.

A bright field TEM micrograph of a typical poly-bump is shown in figure 2, where there is a hint of an embedded particle within the poly-Si layer, capped by two other layers, and a resist mask. In a bright-field TEM image, diffraction contrast is the primary mechanism to enhance the visualization of the particle. However, in a polycrystalline film where there are many grains randomly oriented giving raise to diffraction contrast, a small particle of similar composition and size to the grains will not be easily revealed, which can lead to the conclusion that there is no particle present. One method to overcome this problem is to acquire a primarily inelastic EFTEM image, such as figure 3, at an energy loss where the plasmon tails of the Si-oxide and Si-nitride dominate those of Si.

An analytical imaging technique to characterize an embedded defect such as these poly-bumps is EFTEM spectral imaging where a series of EFTEM images are stacked in energy space giving an EELS spectrum at each image pixel. In the present study, three EFTEM spectral images (SI) were recorded covering the energy loss range from $-5 \mathrm{eV}$ to $650 \mathrm{eV} ;-5 \mathrm{eV}$ to $200 \mathrm{eV}$ in $5 \mathrm{eV}$ steps, $100 \mathrm{eV}$ to $400 \mathrm{eV}$ in $10 \mathrm{eV}$ steps, and $350 \mathrm{eV}$ to $650 \mathrm{eV}$ in $10 \mathrm{eV}$ steps. These spectral images were spatially aligned then spliced together using Gatan's Digital Micrograph (DM) software. The middle and high energy-loss SI's were rebinned in energy to keep the same energy scale as the low energy loss $\mathrm{SI}, 5 \mathrm{eV}$. The full SI was then rotated and cropped to align the substrate to the bottom of the image. Relative composition maps of $\mathrm{Si}, \mathrm{C}, \mathrm{N}$, and $\mathrm{O}$ were extracted from the EFTEM SI dataset, and superimposed in false color as shown in figure 4. One can clearly see that the embedded particle is a Si-oxide particle. The "wings" on either side of the particle are likely due to the particle being

\footnotetext{
${ }^{\dagger}$ CeriumLabs $^{\mathrm{TM}}$ is a trademark of Spansion LLC
} 
cylindrically shaped and sliced at an angle to the cylindrical axis with one "wing" on the top of the TEM lamella and the other "wing" on the bottom.
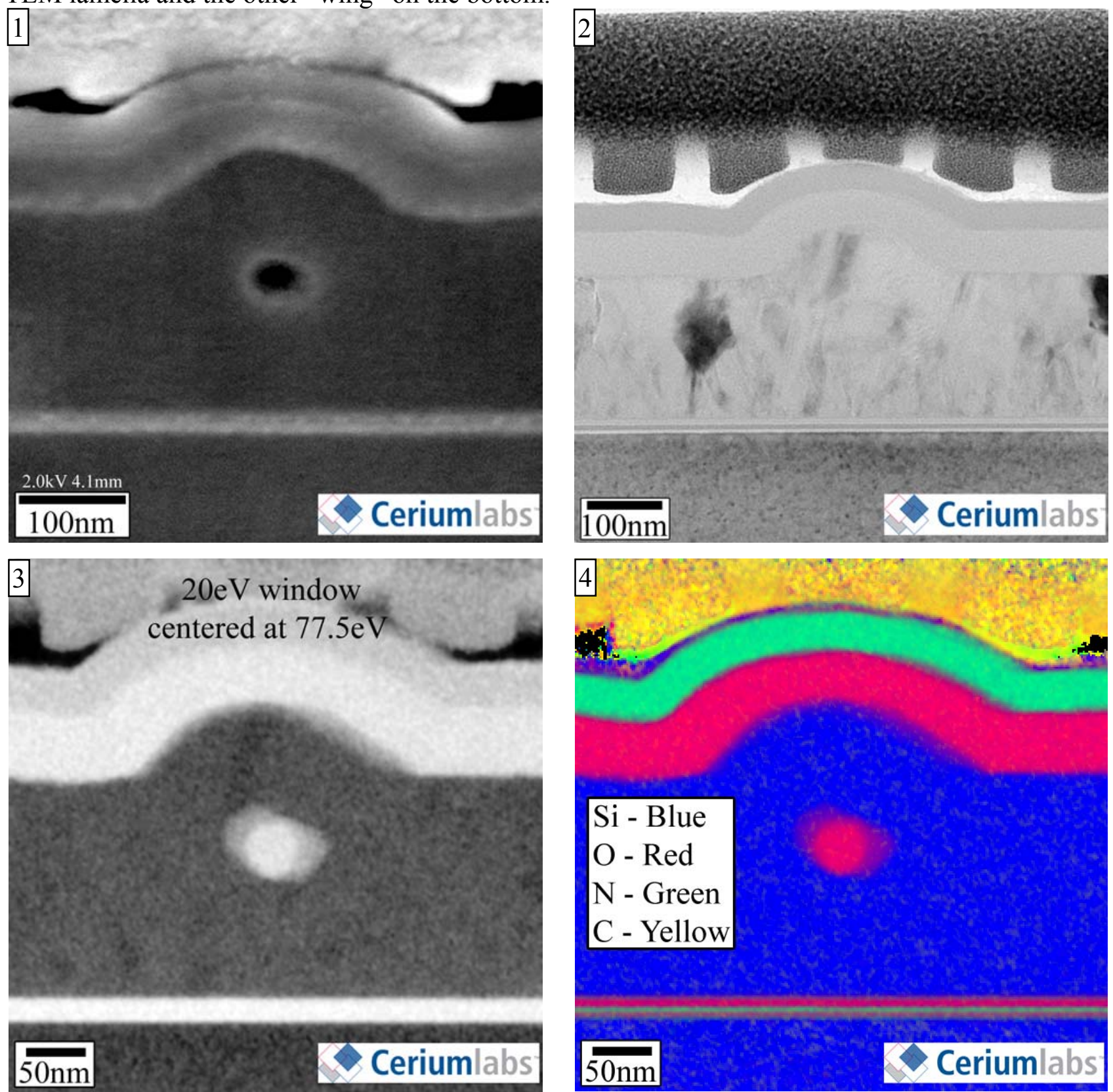

$50 \mathrm{~nm}$

Ceriumlabs

Cross-section SEM image of the defect is shown in figure 1) whereas figure 2) is the TEM micrograph of the same poly bump, but without visualizing a source particle. An EFTEM image in figure 3) enhances the contrast of the particle by using the higher intensity in the Si-oxide and Sinitride Plasmon tails than the surrounding Si. A false color composite image of the EFTEM elemental maps extracted from the EFTEM SI clearly reveals the defect is Si-oxide.

References

[1] S. Wolf and R.N. Tauber, Silicon Processing for the VLSI Era, $2^{\text {nd }}$ ed., Lattice Press, Sunset Beach, 2000

[2] C. Yu et al., ASMC $200617^{\text {th }}$ Annual SEMI/IEEE (2006) 206. 